\title{
Éditorial
}

\section{De Saragosse à Épinal}

Oui, la radioprotection reste perfectible dans toutes ses applications, qu'elles soient industrielles ou médicales. Il y a quelques années, nous avons tous été interpellés par l'accident survenu dans un centre de radiothérapie à Saragosse. Une remédiation ponctuelle à cet événement - singulier, car survenu dans un contexte médical - avait rapidement restauré la confiance nécessaire, celle des malades, celle des professionnels aussi. Et c'est alors que «l'affaire»d'Épinal a été dévoilée ; elle reste l'accident de radiothérapie le plus important jamais déploré à ce jour en France.

Faut-il en conclure que la radiothérapie est dangereuse ?

Bien entendu, non ! La radiothérapie participe au traitement - et à la guérison de centaines de milliers de malades chaque année, mais elle doit être dotée d'exigences aussi strictes que celles mises en œuvre dans d'autres domaines. Elle doit, de la même façon, tirer toutes les leçons des dysfonctionnements, incidents ou accidents, pour garantir les conditions optimales de sécurité et donc de qualité.

Ce cercle vertueux a été initié grâce à l'implication de tous les professionnels concernés, grâce aussi à la rigueur des autorités de sûreté française et espagnole. Ces messages ont été explicités de façon claire et documentée lors du dernier congrès national de la SFRP (Angers) par Léopoldo Arranz et Michel Bourguignon. L'intérêt évident de ces contributions nous a conduit à publier ces deux articles sans délai, afin de leur conserver l'actualité qu'ils méritent. Ces deux articles soulignent bien le rôle important des physiciens médicaux tel que l'avait présenti Daniel Blanc lors de la création du DEA en 1970.

Ce dernier numéro de l'année est également une agréable occasion, celle de vous présenter, au nom du Comité de rédaction, nos meilleurs vœux pour l'année 2010. Nous remercions chaleureusement tous les auteurs qui nous ont envoyé un grand nombre de projets en 2009 ; tous les articles n'ont pas été publiés car nos évaluateurs (dont vous retrouverez la liste en fin de volume) sont vigilants et exigeants pour conserver à la revue le niveau de qualité que notre lectorat attend. Qu'ils en soient tous remerciés et que la belle dynamique de publication perdure !...

Henri Métivier

Président du Comité de rédaction

DOI: $10.1051 /$ radiopro/2009025 